\title{
Partial Synchronizability Characterized by Principal Quasi-Submatrices Corresponding to Clusters
}

\author{
Gang Zhang, ${ }^{1}$ Zhongjun Ma, ${ }^{2}$ Yi Wang, ${ }^{3}$ and Jianbao Zhang ${ }^{4}$ \\ ${ }^{1}$ College of Mathematics and Information Science, Hebei Normal University, Shijiazhuang 050024, China \\ ${ }^{2}$ School of Mathematics and Computing Science, Guilin University of Electronic Technology, Guilin 541004, China \\ ${ }^{3}$ School of Mathematics and Statistics, Zhejiang University of Finance and Economics, Hangzhou 310018, China \\ ${ }^{4}$ School of Science, Hangzhou Dianzi University, Hangzhou 310018, China
}

Correspondence should be addressed to Yi Wang; wangyihzh@gmail.com

Received 17 May 2014; Accepted 3 July 2014; Published 16 July 2014

Academic Editor: Jun $\mathrm{Hu}$

Copyright (C) 2014 Gang Zhang et al. This is an open access article distributed under the Creative Commons Attribution License, which permits unrestricted use, distribution, and reproduction in any medium, provided the original work is properly cited.

\begin{abstract}
A partial synchronization problem in an oscillator network is considered. The concept on a principal quasi-submatrix corresponding to the topology of a cluster is proposed for the first time to study partial synchronization. It is shown that partial synchronization can be realized under the condition depending on the principal quasi-submatrix, but not distinctly depending on the intercluster couplings. Obviously, the dimension of any principal quasi-submatrix is usually far less than the one of the network topology matrix. Therefore, our criterion provides us a novel index of partial synchronizability, which reduces the network size when the network is composed of a great mount of nodes. Numerical simulations are carried out to confirm the validity of the method.
\end{abstract}

\section{Introduction}

Since the pioneering work of Pecora and Carroll [1], extensive researches on chaos synchronization have been carried out due to their potential applications in various disciplines such as physics, engineering, and biology [2-5]. For example, wireless sensor networks are widely researched due to the important applications in the real scenarios. One of the biggest challenges in investigating wireless sensor networks is how to obtain higher synchronization accuracy with minimal overhead [6]. And many other network-induced phenomena have also been discussed extensively in engineering [7] .

Up to date, there have been many synchronization types being proposed and discussed, including complete synchronization $[8,9]$, partial synchronization [10], and inner and outer synchronization [11]. Generally speaking, there exists an intimate relationship between the phenomena of synchronization and invariant manifolds of coupled systems [9-12]. Thus, when we carry out researches on partial synchronization of coupled systems, it is always supposed that the corresponding full or partial synchronization manifolds are invariant manifolds. The established tools for the stability of invariant synchronization manifolds mainly consist of local linearization method and Lyapunov function method. One of the prominent results of the former is the master stability function method [9]. The method has obtained two significant factors determining the local stability of the synchronous state, that is, the maximum Lyapunov exponent of the node dynamics and the eigenvalues of the topology matrix. One of the prominent results of the latter is the study of the global attractiveness of the synchronization manifold. A crucial requirement for the method is the condition of the node dynamics satisfying $f \in \operatorname{QUAD}(\Delta, P, \Omega)$ [10]. In some sense, the condition means that the system can synchronize when the coupling is made sufficiently large. Recently, some new conditions have been obtained for synchronization of networks without Lipschitz condition or QUAD condition [12].

The type of synchronization concerned in this paper is partial synchronization. It means that the coupled oscillators split into subgroups called clusters, and all the oscillators in the same cluster behave in the same fashion. Many relative 
studies have been carried out [13-18]. By using pinning control strategy, partial synchronization of coupled stochastic delayed neural networks was discussed in [14]. Similar control strategy is also proposed to select controlled communities by analyzing the information of each community such as in-degrees and out-degrees [15]. Afterwards, some simple intermittent pinning controls and centralized adaptive intermittent controls are proposed [16]. However, a suitable control law must be presented in order to use pinning control strategy. Some other researches focused on partial synchronization induced by the coupling configuration. An arbitrarily selected partial synchronization manifold was constructed for a network with cooperative and competitive couplings [17]. Recently, a sufficient and necessary condition for partial synchronization manifolds being invariant manifolds was obtained in networks coupled linearly and symmetrically [10]. More significantly, some sufficient conditions for the global attractiveness of the partial synchronization manifold were derived by decomposing the whole space into a direct sum of the synchronization manifold and the transverse space. The results are meaningful and interesting. Based on the method in [10], partial synchronization bifurcations [19] were analyzed for a globally coupled network with a parameter, which topology is not complex. Nevertheless, all the eigenvalues of the topology matrix are essential for that method, which needs a large quantity of computation when the network size is very large.

In this paper, a novel criterion on partial synchronization is proposed through the analysis of principal quasi-submatrices corresponding to the clusters. Previous researches have obtained several criteria on partial synchronization [10]. However, these criteria depend heavily on the topology matrix of the whole network. For many complex networks in real world, it is tedious to obtain the eigenvalues and eigenvectors of the topology matrix of the whole network. Therefore, this paper aims to propose a novel criterion, which is not distinctly dependent on the intercluster couplings and the topology matrix of the whole network. Instead, it is sufficient for partial synchronization to verify that the conditions on the intracluster couplings are satisfied. Therefore, it will be advantageous to discuss partial synchronization in the complex network with a large number of nodes because the dimension of any principal quasi-submatrix is usually far less than the one of the network topology matrix. At first, the tedious work of solving the eigenvectors corresponding to the second-largest eigenvalue is avoided. Secondly, partial synchronization is studied by the inner topologies of the individual clusters. Obviously, the network size reduction provides convenience for the study of partial synchronization in networks with great mounts of oscillators. In order to confirm its effectiveness, some numerical simulations are carried out to study partial synchronization in a star-global network. The numerical simulations are in good agreement with the theoretical analysis.

The present paper is built up as follows. Some concerned concepts and a lemma for the invariance of the partial synchronization manifold are introduced in Section 2. Principal quasi-submatrices corresponding to the clusters are proposed, and our main results are then established in Section 3. Numerical examples are presented to confirm the effectiveness of the results in Section 4. Finally, a brief discussion of the obtained results is given in Section 5 .

\section{Preliminaries}

In this section, we introduce some basic concepts of invariant synchronization manifolds and a related lemma, which are required throughout the paper.

In past years, many studies $[9,10,12]$ of synchronization phenomena focused on oscillator networks coupled linearly and symmetrically. The system can be described by the following ordinary differential equations:

$$
\dot{x}_{i}=f\left(x_{i}, t\right)+\varepsilon \sum_{j=1}^{m} a_{i j} \Gamma x_{j}, \quad i=1, \ldots, m,
$$

where $x_{i}=\left(x_{i}^{1}, \ldots, x_{i}^{n}\right)^{\top}$ is the $n$-dimensional state variable of the $i$ th oscillator, $m>1$ is the network size, $t \in[0,+\infty)$ is a continuous time, $f: R^{n} \times[0,+\infty) \rightarrow R^{n}$ is a continuous map, $\varepsilon>0$ is the coupling strength, and $\Gamma=\operatorname{diag}\left\{\gamma_{1}, \ldots\right.$, $\left.\gamma_{n}\right\}$ is a nonnegative matrix determining the interaction of variables. The coupling weight matrix $A=\left(a_{i j}\right)_{m \times m}$ is assumed to satisfy that $a_{i j}=a_{j i} \geq 0$, for $i \neq j$, and $\sum_{j=1}^{m} a_{i j}=$ $s$ for $i=1, \ldots, m$.

In order to study partial synchronization of the system (1), the set of nodes $\{1, \ldots, m\}$ is divided into $d$ nonempty subsets (clusters). Let $G=\left\{G^{1}, \ldots, G^{d}\right\}$ be the partition, and denote $K=\left(K_{1}, K_{2}, \ldots, K_{d}\right)$, where $K_{k} \geq 1$ is the cardinal number of the cluster $G^{k}, k=1, \ldots, d$. Without loss of generality, suppose that $G^{1}=\left\{1, \ldots, K_{1}\right\}, \ldots, G^{d}=$ $\left\{\sum_{p=1}^{d-1} K_{p}+1, \ldots, m\right\}$. Based on the partition $G$, we rewrite the coupling matrix $A$ as a block matrix,

$$
A=\left[\begin{array}{cccc}
A_{11} & A_{12} & \cdots & A_{1 d} \\
A_{21} & A_{22} & \cdots & A_{2 d} \\
\cdots & \cdots & \cdots & \cdots \\
A_{d 1} & A_{d 2} & \cdots & A_{d d}
\end{array}\right],
$$

where $A_{k l} \in R^{K_{k} \times K_{l}}, k, l=1, \ldots, d$.

We will discuss sufficient conditions for the $K_{k}$ nodes in the cluster $G^{k}$ to synchronize with each other, $k=1, \ldots, d$. Before that, the concepts of partial synchronization manifolds and transverse spaces introduced in the following [10]:

$$
\begin{gathered}
\mathbb{M}_{K}=\left\{\left(x_{1}^{\top}, \ldots, x_{m}^{\top}\right)^{\top} \mid\left(x_{\sum_{p=0}^{k-1} K_{p}+1}^{\top}, \ldots, x_{\sum_{p=0}^{k} K_{p}}^{\top}\right)^{\top} \in \mathbb{M}_{K}^{k},\right. \\
k=1, \ldots, d\},
\end{gathered}
$$

where $K_{0}=0$,

$$
\begin{aligned}
\mathbb{M}_{K}^{k}=\left\{\left(x_{\sum_{p=0}^{k-1} K_{p}+1}^{\top}, \ldots, x_{\sum_{p=0}^{k} K_{p}}^{\top}\right)^{\top}\right. & \\
& \left.\in R^{n K_{k}} \mid x_{\sum_{p=0}^{k-1} K_{p}+1}=\cdots=x_{\sum_{p=0}^{k} K_{p}}\right\},
\end{aligned}
$$


are called a partial synchronization manifold. We also call

$$
\begin{gathered}
\mathbb{L}_{K}=\left\{\left(x_{1}^{\top}, \ldots, x_{m}^{\top}\right)^{\top} \mid\left(x_{\sum_{p=0}^{k-1} K_{p}+1}^{\top}, \ldots, x_{\sum_{p=0}^{k} K_{p}}^{\top}\right)^{\top} \in \mathbb{L}_{K}^{k},\right. \\
k=1, \ldots, d\},
\end{gathered}
$$

where

$$
\begin{array}{r}
\mathbb{L}_{K}^{k}=\left\{\left(x_{\sum_{p=0}^{k-1} K_{p}+1}^{\top}, \ldots, x_{\sum_{p=0}^{k} K_{p}}^{\top}\right)^{\top}\right. \\
\left.\in R^{n K_{k}} \mid \sum_{i=1}^{K_{k}} x_{\sum_{p=0}^{k-1} K_{p}+i}=0\right\},
\end{array}
$$

a transverse space for $\mathbb{M}_{K}$.

In case $n=1$, the four sets mentioned above are denoted by $M_{K}, M_{K}^{k}, L_{K}$, and $L_{K}^{k}$, respectively. In case $d=$ 1 , the synchronization manifold $\mathbb{M}_{K}$ is called a full synchronization manifold. For simplicity, we denote the full synchronization manifold $\mathbb{M}_{K}$ by $\mathbb{M}$, and the corresponding transverse space $\mathbb{L}_{K}$ by $\mathbb{L}$. Sometimes, the manifold $\mathbb{M}_{K}$ is also denoted by $\mathbb{M}_{K}(G)$ to emphasize its partition $G$.

Definition 1. The synchronization manifold $\mathbb{M}_{K}$ is said to be globally attractive for the system (1), or the system (1) is said to realize partial synchronization with the partition $G=\left\{G^{1}, G^{2}, \ldots, G^{d}\right\}$ if, for any initial condition $\left(x_{1}^{\top}(0)\right.$, $\left.x_{2}^{\top}(0), \ldots, x_{m}^{\top}(0)\right)^{\top} \in R^{m n}$,

$$
\lim _{t \rightarrow+\infty} \sum_{k=1}^{d} \sum_{i \in G^{k}}\left\|x_{i}(t)-x_{\sum_{p=0}^{k-1} K_{p}+1}(t)\right\|=0,
$$

where $\|\cdot\|$ denotes 2 -norm of vectors. In case of $d=1$, the system (1) is said to realize full synchronization, if the full synchronization manifold $\mathbb{M}$ is globally attractive.

Synchronization manifolds are always supposed to be invariant in order to discuss the attractiveness. Therefore, it is necessary to recall the definition of an invariant manifold [20] for the ordinary differential equations,

$$
\dot{x}=X(x, t), \quad x \in R^{N}, X: R^{N} \times[0,+\infty) \longrightarrow R^{N} .
$$

Denote $\mathscr{M}$ as the manifold of codimension $p$ defined by a vector equation $H(x)=0, H=\left(h_{1}, h_{2}, \ldots, h_{p}\right), 1 \leq p \leq$ $N-1 . \mathscr{M}$ is called an invariant manifold of the system (8) if

$$
\left.(\operatorname{grad} H \cdot X)\right|_{H=0} \equiv 0,
$$

which implies that the vector field (8) is tangent to $\mathscr{M}$. For more details of the existence of invariant manifolds, one can refer to the papers by Golubitsky and coworkers [21, 22]. The following lemma gives a sufficient and necessary condition for a partial synchronization manifold being an invariant manifold.

Lemma 2 (see [10]). Let $K=\left(K_{1}, K_{2}, \ldots, K_{d}\right)$. The synchronization manifold $\mathbb{M}_{K}$ is an invariant manifold of the system (1) if and only if the coupling matrix $A$ has the form (2) with every $A_{i j}$ being equal row sum.
Remark 3. Based on Lemma 2, we can find all invariant synchronization manifolds for a given coupling matrix. As we know, each diagonal block reveals the intracluster information communication, and each nondiagonal block represents the information communication among different clusters. By the condition that every $A_{i j}$ is equal row sum, we mean that every node in the same cluster receive an equal amount of information communication from every other cluster.

\section{Main Results}

Noticing the sufficient and necessary condition in Lemma 2, we assume that every submatrix $A_{k l} \in R^{K_{k} \times K_{l}}$ is an equal row sum matrix with row sum $s_{k l}, k, l=1, \ldots, d$. Since $A^{s}$ is an equal row sum matrix, that is, $\sum_{j=1}^{m} a_{i j}=\sum_{k=1}^{d} s_{\hat{i} \hat{k}}=s$, where the denotation $\hat{i}$ represents $k$, for all $i \in G^{k}$, we define the matrices $\bar{A}_{k k}=\left(\bar{a}_{i j}\right)_{K_{k} \times K_{k}}$ as

$$
\bar{A}_{k k}=A_{k k}+\left(s-s_{k k}\right) E_{K_{k}}=A_{k k}+\sum_{l=1, l \neq k}^{d} s_{k l} E_{K_{k}},
$$

where $E_{K_{k}} \in R^{K_{k} \times K_{\mathrm{k}}}$ is the identity matrix, $k=1, \ldots, d$. It is easy to conclude from (10) that $\sum_{j \in G^{i}} \bar{a}_{i j}=s$ and

$$
\bar{a}_{i j}= \begin{cases}a_{i j}, & i \neq j \\ a_{i j}+\sum_{l=1, l \neq \hat{i}}^{d} s_{\hat{i}}, & i=j .\end{cases}
$$

Noticing that $A_{k k}$ is a principal submatrix of $A$, we call the matrix $\bar{A}_{k k}$ a principal quasi-submatrix corresponding to the cluster $G^{k}, k=1, \ldots, d$.

Now, we are now in a position to carry out the following theorem with the help of Lyapunov function method.

Theorem 4. Let $K=\left(K_{1}, K_{2}, \ldots, K_{d}\right), P=\operatorname{diag}\left(p_{1}, \ldots, p_{n}\right)$ be a positive-definite diagonal matrix, and let $\Delta=$ $\operatorname{diag}\left(\delta_{1}, \ldots, \delta_{n}\right)$ be a diagonal matrix. Suppose $\delta_{j} \leq 0$ if $j \notin J$, where $J=\left\{j: 1 \leq j \leq n, \gamma_{j} \neq 0\right\}$. Then under the following three conditions.

(i) Every submatrix $A_{k l}$ in the block matrix (2) has equal row sum $s_{k l}$, and every principal submatrix $A_{k k}$ is irreducible.

(ii) There exists a constant $\epsilon>0$ such that, for any $u, v \in R^{n}$ and all $t \geq 0$,

$$
\begin{gathered}
(u-v)^{\top} P\{[f(u, t)-f(v, t)]-\Delta(u-v)\} \\
\leq-\epsilon(u-v)^{\top}(u-v) .
\end{gathered}
$$

(iii) For all $j=1, \ldots, n, k=1, \ldots, d$, the matrices $\varepsilon \gamma_{j} \bar{A}_{k k}+$ $\delta_{j} E_{K_{k}}$ are negative semidefinite in the transverse space $L_{K}^{k}$, that is,

$$
z^{\top}\left(\varepsilon \gamma_{j} \bar{A}_{k k}+\delta_{j} E_{K_{k}}\right) z \leq 0, \quad z \in L_{K}^{k}
$$


or, in particular,

$$
\varepsilon \gamma_{j} \lambda_{k}^{(2)}+\delta_{j} \leq 0
$$

where $\lambda_{k}^{(2)}$ is the second-largest eigenvalue of $\bar{A}_{k k}$.

The synchronization manifold $\mathbb{M}_{K}$ is globally attractive for the system (1).

For convenience of the proof, the following notations are introduced.

$$
\begin{gathered}
\bar{x}_{k}(t)=\frac{1}{K_{k}} \sum_{i \in G^{k}} x_{i}(t), \quad \bar{x}(t)=\left[\bar{x}_{\widehat{1}}^{\top}(t), \ldots, \bar{x}_{\widehat{m}}^{\top}(t)\right]^{\top}, \\
\delta x_{i}(t)=x_{i}(t)-\bar{x}_{\hat{i}}(t), \\
\delta x(t)=\left[\delta x_{1}^{\top}(t), \ldots, \delta x_{m}^{\top}(t)\right]^{\top}, \\
\delta \tilde{x}_{k}^{s}(t)=\left[\delta x_{\sum_{p=0}^{s-1} K_{p}+1}^{s}(t), \ldots, \delta x_{\sum_{p=0}^{s} K_{p}}(t)\right]^{\top}, \\
\delta \tilde{x}_{k}(t)=\left[\delta \tilde{x}_{k}^{1 \top}(t), \ldots, \tilde{x}_{k}^{n \top}(t)\right]^{\top},
\end{gathered}
$$

where $k=1, \ldots, d, i=1, \ldots, m, s=1, \ldots, n$.

Then any vector $x=\left(x_{1}^{\top}, \ldots, x_{m}^{\top}\right)^{\top} \in R^{m n}$ can be decomposed into

$$
x=\bar{x} \oplus \delta x, \quad \bar{x} \in \mathbb{M}_{K}, \quad \delta x \in \mathbb{L}_{K} .
$$

Therefore, the attractiveness of the invariant synchronization manifold $\mathbb{M}_{K}$ is equivalent to $\delta x \rightarrow 0$ when $t \rightarrow+\infty$; that is, the dynamical flow in the transverse subspace $\mathbb{L}_{K}$ converges to zero.

Proof. Noticing the condition (i), one gets

$$
\begin{aligned}
\sum_{j=1}^{m} a_{i j} \Gamma x_{j}(t) & =\sum_{l=1}^{d} \sum_{j \in G^{l}} a_{i j} \Gamma\left[\delta x_{j}(t)+\bar{x}_{l}(t)\right] \\
& =\sum_{l=1}^{m} a_{i j} \Gamma \delta x_{j}(t)+\sum_{l=1}^{d} s_{\widehat{i l}} \Gamma \bar{x}_{l}(t) .
\end{aligned}
$$

Therefore,

$$
\begin{aligned}
\frac{d \delta x_{i}(t)}{d t} & =\frac{d x_{i}(t)}{d t}-\frac{1}{K_{\hat{i}}} \sum_{p \in G^{\hat{i}}} \frac{d x_{p}(t)}{d t} \\
& =f\left(x_{i}(t), t\right)-f\left(\bar{x}_{\hat{i}}(t), t\right)+\varepsilon \sum_{j=1}^{m} a_{i j} \Gamma \delta x_{j}(t)+J_{\hat{i}},
\end{aligned}
$$

where

$$
\begin{aligned}
J_{\widehat{i}}= & f\left(\bar{x}_{\hat{i}}(t), t\right)-\frac{1}{K_{\hat{i}}} \sum_{p \in G^{\hat{j}}}\left[f\left(x_{p}(t), t\right)+\varepsilon \sum_{q=1}^{m} a_{p q} \Gamma x_{q}(t)\right] \\
& +\varepsilon \sum_{l=1}^{d} s_{\hat{i} l} \Gamma \bar{x}_{l}(t) .
\end{aligned}
$$

In order to utilize the $\operatorname{QUAD}\left(\Delta, P, R^{n}\right)$ condition, a Lyapunov function is defined as follows:

$$
V(\delta x(t))=\frac{1}{2} \sum_{j=1}^{m} \delta x_{i}^{\top}(t) P \delta x_{i}(t) .
$$

One can conclude from $\sum_{i \in G^{k}} \delta x_{i}(t)=0$ that

$$
\sum_{i=1}^{m} \delta x_{i}^{\top}(t) P J_{\hat{i}}=\sum_{k=1}^{d}\left[\sum_{i \in G^{k}} \delta x_{i}^{\top}(t)\right] P J_{\hat{i}}=0,
$$

and then,

$$
\begin{aligned}
& \frac{d V\left(\delta x_{i}(t)\right)}{d t} \\
& =\sum_{i=1}^{m} \delta x_{i}^{\top}(t) P\left[f\left(x_{i}(t), t\right)-f\left(\bar{x}_{i}(t), t\right)+\varepsilon \sum_{j=1}^{m} a_{i j} \Gamma \delta x_{j}(t)\right] \\
& \leq-\epsilon \sum_{i=1}^{m} \delta x_{i}^{\top}(t) \delta x_{i}(t) \\
& +\sum_{i=1}^{m} \delta x_{i}^{\top}(t) P\left[\varepsilon \sum_{j=1}^{m} a_{i j} \Gamma \delta x_{j}(t)+\Delta \delta x_{i}(t)\right] .
\end{aligned}
$$

Denote the second term in the right hand of (22) as $S$ for convenience; then,

$$
\begin{array}{r}
S=\sum_{k=1}^{d} \sum_{i \in G^{k}} \delta x_{i}^{\top}(t) P\left[\varepsilon \sum_{l=1}^{d} \sum_{j \in G^{l}} a_{i j} \Gamma \delta x_{j}(t)+\Delta \delta x_{i}(t)\right] \\
=\sum_{k=1}^{d} \sum_{i \in G^{k}} \delta x_{i}^{\top}(t) P\left[\varepsilon \sum_{j \in G^{k}} a_{i j} \Gamma \delta x_{j}(t)+\Delta \delta x_{i}(t)\right. \\
\left.+\varepsilon \sum_{l=1, l \neq k}^{d} \sum_{j \in G^{l}} a_{i j} \Gamma \delta x_{j}(t)\right]
\end{array}
$$

Since $i, j \in G^{k}$ holds for the first term in the right hand above, replacing $a_{i j}$ with $\bar{a}_{i j}$ according to (11) gives rise to that

$$
\begin{aligned}
S= & \sum_{k=1}^{d} \sum_{i \in G^{k}} \delta x_{i}^{\top}(t) P\left[\varepsilon \sum_{j \in G^{k}} \bar{a}_{i j} \Gamma \delta x_{j}(t)+\Delta \delta x_{i}(t)\right] \\
& +\varepsilon \sum_{k=1}^{d} \sum_{i \in G^{k}} \delta x_{i}^{\top}(t) P \sum_{l=1, l \neq \hat{i}}^{d}\left[-s_{\hat{i} l} \Gamma \delta x_{i}(t)+\sum_{j \in G^{l}} a_{i j} \Gamma \delta x_{j}(t)\right] \\
= & S_{1}+S_{2} .
\end{aligned}
$$


As a result of the condition (13), one obtains that

$$
\begin{aligned}
S_{1} & =\sum_{k=1}^{d} \sum_{i \in G^{k}} \delta x_{i}^{\top}(t) P\left[\varepsilon \sum_{j \in G^{k}} \bar{a}_{i j} \Gamma \delta x_{j}(t)+\Delta \delta x_{i}(t)\right] \\
& =\sum_{k=1}^{d} \sum_{s=1}^{n} p_{s} \delta \tilde{x}_{k}^{s \top}(t)\left(\varepsilon \gamma_{s} \bar{A}_{k k}+\delta_{s} E_{K_{k}}\right) \delta \tilde{x}_{k}^{s}(t) \leq 0 .
\end{aligned}
$$

Since $s_{\hat{i}}=\sum_{j \in G^{l}} a_{i j}$, the sum $S_{2}$ can be decomposed into

$$
\begin{aligned}
S_{2}= & \varepsilon \sum_{k=1}^{d} \sum_{i \in G^{k}} \delta x_{i}^{\top}(t) P \sum_{l=1, l \neq k}^{d} \sum_{j \in G^{l}} a_{i j} \Gamma\left(\delta x_{j}(t)-\delta x_{i}(t)\right) \\
= & \varepsilon \sum_{k=1}^{d-1} \sum_{l=k+1}^{d} \sum_{i \in G^{k}} \sum_{j \in G^{l}} a_{i j} \delta x_{i}^{\top}(t) P \Gamma\left(\delta x_{j}(t)-\delta x_{i}(t)\right) \\
& +\varepsilon \sum_{l=1}^{d-1} \sum_{k=l+1}^{d} \sum_{i \in G^{k}} \sum_{j \in G^{l}} a_{i j} \delta x_{i}^{\top}(t) P \Gamma\left(\delta x_{j}(t)-\delta x_{i}(t)\right) .
\end{aligned}
$$

Renaming in the second term $k$ by $l, i$ by $j$, and vice versa [23] and utilizing the symmetry of $a_{i j}$, or $A_{l k}^{\top}=A_{k l}$, one gets

$$
\begin{array}{r}
S_{2}=\varepsilon \sum_{k=1}^{d-1} \sum_{l=k+1}^{d} \sum_{i \in G^{k}} \sum_{j \in G^{l}} a_{i j} \delta x_{i}^{\top}(t) P \Gamma\left(\delta x_{j}(t)-\delta x_{i}(t)\right) \\
+\varepsilon \sum_{k=1}^{d-1} \sum_{l=k+1}^{d} \sum_{j \in G^{l}} \sum_{i \in G^{k}} a_{j i} \delta x_{j}^{\top}(t) P \Gamma\left(\delta x_{i}(t)-\delta x_{j}(t)\right) \\
=-\varepsilon \sum_{k=1}^{d-1} \sum_{l=k+1}^{\mathrm{d}} \sum_{i \in G^{k}} \sum_{j \in G^{l}} a_{i j}\left(\delta x_{j}(t)-\delta x_{i}(t)\right)^{\top} P \Gamma \\
\times\left(\delta x_{j}(t)-\delta x_{i}(t)\right)
\end{array}
$$

$\leq 0$

Therefore, one obtains that $S \leq 0$ and

$$
\frac{d V\left(\delta x_{i}(t)\right)}{d t} \leq-\epsilon \sum_{i=1}^{m} \delta x_{i}^{\top}(t) \delta x_{i}(t) \leq-2 \epsilon \frac{V\left(\delta x_{i}(t)\right)}{\max _{i} p_{i}}
$$

which implies that the partial synchronization manifold $\mathbb{M}_{K}$ is globally attractive for the system (1).

The remainder of the proof is to show that condition (14) is also sufficient for $S_{1} \leq 0$.

It is well known that a symmetric matrix $\bar{A}_{k k}$ has the decomposition $\bar{A}_{k k}=U_{k} \Lambda_{k} U_{k}^{\top}$, where $\Lambda_{k}=\operatorname{diag}\left\{\lambda_{k}^{(1)}, \ldots\right.$, $\left.\lambda_{k}^{\left(K_{k}\right)}\right\}$ is a real diagonal matrix and $U_{k} \in R^{K_{k} \times K_{k}}$ is an unitary matrix, that is, $U_{k} U_{k}^{\top}=E_{K_{k}}$. The diagonal elements of $\Lambda_{k}$ are the eigenvalues of $\bar{A}_{k k}$ satisfying $s=\lambda_{k}^{(1)}>\lambda_{k}^{(2)} \geq \cdots \geq \lambda_{k}^{\left(K_{k}\right)}$. The $i$ th column of $U_{k}$ is the eigenvector of $\bar{A}_{k k}$ corresponding to the eigenvalue $\lambda_{k}^{(i)}, i=1, \ldots, K_{k}$. By changes of variables $\delta \tilde{x}_{k}^{s}(t)=U \eta_{k}^{s}(t)$, the quadratic form (25) can be diagonalized as follows:

$$
\begin{aligned}
S_{1} & =\sum_{k=1}^{d} \sum_{s=1}^{n} p_{s} \delta \widetilde{x}_{k}^{s \top}(t)\left(\varepsilon \gamma_{s} \bar{A}_{k k}+\delta_{s} E_{K_{k}}\right) \delta \widetilde{x}_{k}^{s}(t) \\
& =\sum_{k=1}^{d} \sum_{s=1}^{n} p_{s} \eta_{k}^{s \top}(t)\left(\varepsilon \gamma_{s} \Lambda_{k}+\delta_{s} E_{K_{k}}\right) \eta_{k}^{s}(t) .
\end{aligned}
$$

Noticing that the first column of $U_{k}$ is $[1, \ldots, 1]^{\top}$, one can conclude from $\sum_{i \in G^{k}} \delta x_{i}(t)=0$ and $\eta_{k}^{s}(t)=U^{\top} \delta \widetilde{x}_{k}^{s}(t)$ that $\eta_{k}^{1}(t)=0$. Therefore, condition (14) is sufficient for $S_{1} \leq 0$.

The proof is completed.

Compared with the previous results [10], the conditions in Theorem 4 are not dependent on the intercluster couplings, which are eliminated in the proof through a set of mathematical skills in inequalities (25) and (27). The obtained results greatly reduce the network sizes theoretically. However, another question arises naturally: how to implement the proposed condition in reality? The following remarks might answer these questions.

Remark 5. In case that the network size is not very large and the coupling matrix is given, it is easy to verify all the conditions in Theorem 4 . In case that the network consists of great mounts of oscillators, it should still be full of challenges to implement though our result reduces the network size greatly. And it might be hard to implement.

In order to make clear the implications of Theorem 4, several remarks on the conditions are given as follows.

Remark 6. (1) Many chaotic oscillators have been proved to satisfy condition (ii), such as Chua circuits [24] and standard Hopfield neural networks [25]. However, many other systems are not the case such as a lattice of $x$-coupled Rössler systems, in which the stability of synchronization regime is lost with the increasing of coupling [26].

(2) Providing that the trajectories of the uncoupled systems $x_{i}=f\left(x_{i}, t\right), i=1, \ldots, m$ are eventually dissipative, that is, the trajectories will be in the absorbing domain $\mathscr{B}$ eventually, it has been proved that each trajectory of the coupled system (1) is also eventually dissipative and will be in the absorbing domain $\underbrace{\mathscr{B} \times \cdots \times \mathscr{B}}_{m}$ [17] eventually. Therefore, condition (ii) holds when the time $t$ is large enough. For example, the uncoupled Lorenz system

$$
\begin{array}{cc}
\dot{u}=\sigma(v-u), & \sigma=10, \\
\dot{v}=r u-v-u w, & r=28, \\
\dot{w}=-b w+u v, & b=\frac{8}{3},
\end{array}
$$

is eventually dissipative, where

$$
\mathscr{B}=\left\{(u)^{2}+(v)^{2}+(w-\sigma-r)^{2}<\frac{b^{2}(\sigma+r)^{2}}{4(b-1)}=B\right\} .
$$


It has been proved that $x$-coupled [27] or $y$-coupled Lorenz systems [28] satisfy condition (ii) when the time $t$ is large enough.

(3) If there exists a $k_{0} \in\{1,2, \ldots, d\}$ such that $K_{k_{0}}=1$, which implies that the subset $G^{k_{0}}$ contains only one element, which does not synchronize with any other node. Without loss of generality, suppose that $K_{k}=1$ for $k>\bar{d}$. In this case, the corresponding principal quasi-submatrix $\bar{A}_{k k}=s$ and the constant $s$ can be regarded as the single eigenvalue of $\bar{A}_{k k}$ since $\bar{A}_{k k} \vec{v}=s \vec{v}$ for any $\vec{v} \in R$. Therefore, the secondlargest eigenvalue $\lambda_{k}^{(2)}$ does not exist. But notice the definition of partial synchronization in Section 2, synchronization in the cluster $G^{k}$ always occurs, and conditions (13) and (14) should be regarded to hold for any $\varepsilon>0, k>\bar{d}$.

(4) Since $A_{k k}$ is irreducible and diffusive, the largest eigenvalue of $A_{k k}$ is zero, which is simple. Therefore, the largest eigenvalue of $\bar{A}_{k k}$ is $s$, and it is simple also. In case $s=$ 0 , it can be seen that $\lambda_{k}^{(2)}<0$ and condition (14) is equivalent to

$$
\varepsilon \geq \frac{\max _{j \in J}\left\{0, \delta_{j} / \gamma_{j}\right\}}{\min _{1 \leq k \leq \bar{d}}\left|\lambda_{k}^{(2)}\right|}
$$

Condition (14) provides us a novel index of partial synchronizability.

\section{Numerical Examples}

Consider the system (1) with $2 m y$-coupled Lorenz systems

$$
\dot{x}_{i}=f\left(x_{i}, t\right)+\varepsilon \sum_{j=1}^{2 m} a_{i j}(\theta) \Gamma x_{j}, \quad i=1, \ldots, 2 m,
$$

where $f\left(x_{i}, t\right), x_{i}=\left(u_{i}, v_{i}, w_{i}\right)^{\top}$ defined by the system (30), $\Gamma=\operatorname{diag}(0,1,0)$. Let $P=\operatorname{diag}(B / 2(b-\epsilon) \sigma+\epsilon / \sigma, 1,1)$, and $\Delta=\operatorname{diag}\left(0,2 b-\epsilon-1+[B-2(b-\epsilon)(\sigma-\epsilon)]^{2} / 2 B(b-\epsilon), 0\right)$, and the $f$ in (27) has been proved to satisfy condition (ii) when the time $t$ is large enough. Through translating time, the network system (27) satisfies condition (ii) in Theorem 4.

4.1. A Star-Global Network. Design the following topology matrix of the system (33) as

$$
A(\theta)=\left[\begin{array}{cc}
A_{11}(\theta) & \theta E_{m} \\
\theta E_{m} & A_{22}(\theta)
\end{array}\right]_{2 m \times 2 m},
$$

where $\theta \geq 0$ is the coupling weight parameter of couplings between the two clusters, and the identity matrix $E_{m}$ implies that the $i$ th $(1 \leq i \leq m)$ oscillator in the first cluster is coupled with the $(m+i)$ th oscillator in the second cluster. As

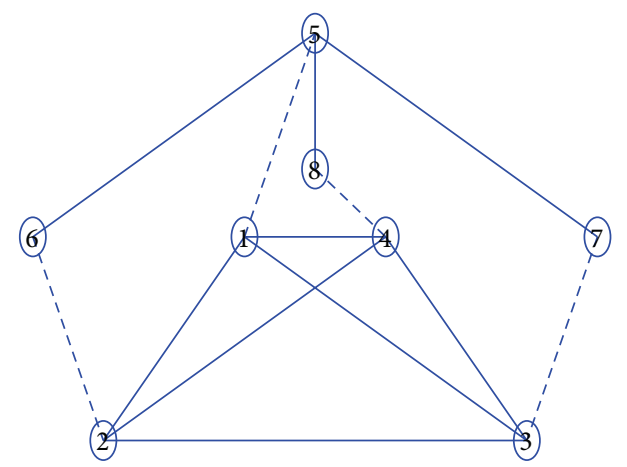

FIGURE 1: Topology structure of a star-global network composed of 8 oscillators. The solid lines represent the inner couplings of each clusters; the dashed lines represent the outer couplings between different clusters.

a special case, the submatrices $A_{11}(\theta)$ and $A_{22}(\theta)$ are taken as follows:

$$
\begin{aligned}
A_{11}(\theta) & =\left[\begin{array}{cccc}
1-m-\theta & 1 & \cdots & 1 \\
1 & 1-m-\theta & \cdots & 1 \\
& \cdots & \cdots & \\
1 & 1 & \cdots & 1-m-\theta
\end{array}\right], \\
A_{22}(\theta) & =\left[\begin{array}{ccccc}
1-m-\theta & 1 & 1 & \cdots & 1 \\
1 & -1-\theta & 0 & \cdots & 0 \\
& \cdots & \cdots & & \\
1 & 0 & 0 & \cdots & -1-\theta
\end{array}\right] .
\end{aligned}
$$

Obviously, $A_{11}(\theta)$ and $A_{22}(\theta)$ are the topology matrices of a globally coupled network and a star-coupled network, respectively. Therefore, we call the network a star-global (coupled) network.

4.2. Numerical Simulations. As an example, the topology structure of a star-global network with $m=4$ is shown in Figure 1. Due to the specific topological structure of the network, the following partitions satisfy condition (i),

$$
\begin{aligned}
& G_{1}=\{1,2,3,4,5,6,7,8\}, \\
& G_{2}=\{1,2,3,4 ; 5,6,7,8\}, \\
& G_{3}=\{1,5 ; 2,3,4,6,7,8\} .
\end{aligned}
$$

Remark 7. According to the criterion in the previous researches [10], we should firstly find the eigenvalues and the corresponding eigenvectors of the $8 \times 8$ matrix $A(\theta)$. As we know, it should be of a vast amount of calculations. However, based on Theorem 4, it is enough for partial synchronization with the partition $G_{2}\left(G_{3}\right)$ if we can find the eigenvalues of two $4 \times 4$ matrices (four $2 \times 2$ matrices). Obviously, Theorem 4 reduces the calculations greatly.

The principal quasi-submatrices corresponding to the clusters in the partitions $G_{1}$ or $G_{2}$, are $A(\theta)$ or $\left.A_{11}(\theta)\right|_{\theta=0}$ and $\left.A_{22}(\theta)\right|_{\theta=0}$, respectively. And denote the ones in the partition 


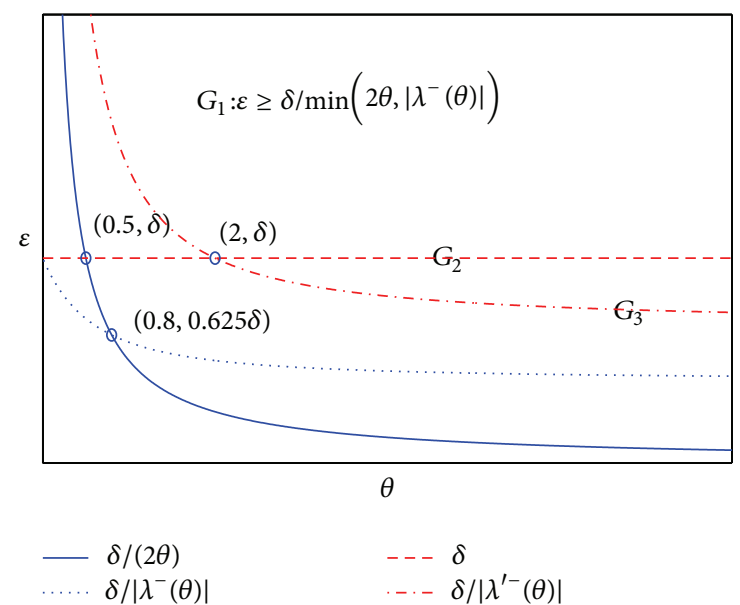

FIGURE 2: Threshold of the coupling strength $\varepsilon$ and the coupling weight $\theta$ for partial synchronization with different partitions.

$G_{3}$ as $\bar{A}_{11}(\theta)$ and $\bar{A}_{22}(\theta)$. Further analysis gives rise to the eigenvalues sets of the quasi-submatrices,

$$
\begin{gathered}
\sigma(A(\theta))=\left\{0,-4,-2 \theta,-4-2 \theta, \lambda^{ \pm}, \lambda^{ \pm}\right\}, \\
\sigma\left(\left.A_{11}(\theta)\right|_{\theta=0}\right)=\{0,-4,-4,-4\}, \\
\sigma\left(\left.A_{22}(\theta)\right|_{\theta=0}\right)=\{0,-1,-1,-4\}, \\
\sigma\left(\bar{A}_{11}(\theta)\right)=\{0,-2 \theta\}, \\
\sigma\left(\bar{A}_{22}(\theta)\right)=\left\{0,-2 \theta, \lambda^{\prime \pm}, \lambda^{\prime \pm}\right\},
\end{gathered}
$$

where $\lambda^{ \pm}=-\left(2 \theta+5 \pm \sqrt{9+4 \theta^{2}}\right) / 2, \lambda^{\prime \pm}=-(2 \theta+$ $\left.3 \pm \sqrt{9+4 \theta^{2}}\right) / 2$. Therefore, partial synchronization with the partition $G_{2}$ occurs if $\varepsilon \geq \delta$; partial synchronization with the partition $G_{3}$ occurs if $\varepsilon \geq \delta /\left|\lambda^{\prime-}(\theta)\right|$; and full synchronization with the partition $G_{1}$ occurs if $\varepsilon \geq \max \left\{\delta / 2 \theta, \delta /\left|\lambda^{-}(\theta)\right|\right\}$. These are seen much more clearly in Figure 2 .

By fixing $\theta \in(0,0.5]$ and increasing $\varepsilon$ gradually, partial synchronization with the partition $G_{2}$ will firstly occur; then, the one with $G_{3}$ and full synchronization occurs at the same time. Figure 2 also implies that the threshold for partial synchronization with the partition $G_{3}$ is sufficient for full synchronization.

In order to validate the effectiveness of Figure 2 numerically, the following average cluster errors of the system (33) are defined to measure partial synchronization with partitions $G_{1}, G_{2}$, and $G_{3}$, respectively,

$$
\begin{gathered}
e_{1}=\frac{1}{8} \sum_{i=1}^{8}\left\|x_{i}\left(t_{0}\right)-x_{1}\left(t_{0}\right)\right\|, \\
e_{2}=\frac{1}{8} \sum_{i=1}^{4}\left\|x_{i}\left(t_{0}\right)-x_{1}\left(t_{0}\right)\right\|+\frac{1}{8} \sum_{i=5}^{8}\left\|x_{i}\left(t_{0}\right)-x_{5}\left(t_{0}\right)\right\|, \\
e_{3}=\frac{1}{8}\left\|x_{5}\left(t_{0}\right)-x_{1}\left(t_{0}\right)\right\| \\
+\frac{1}{8} \sum_{i=2, i \neq 5}^{8}\left\|x_{i}\left(t_{0}\right)-x_{2}\left(t_{0}\right)\right\| .
\end{gathered}
$$

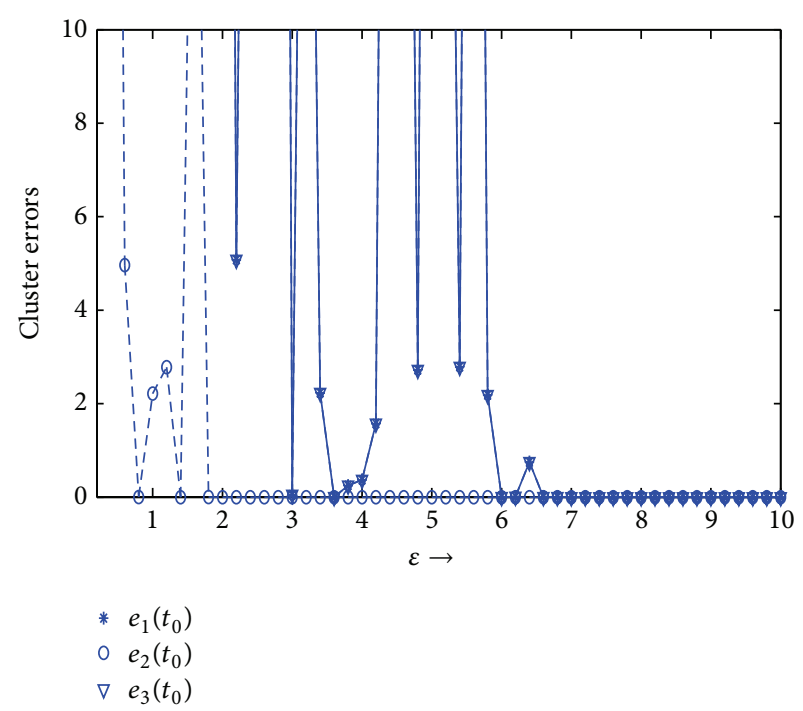

(a) $\theta=0.2$

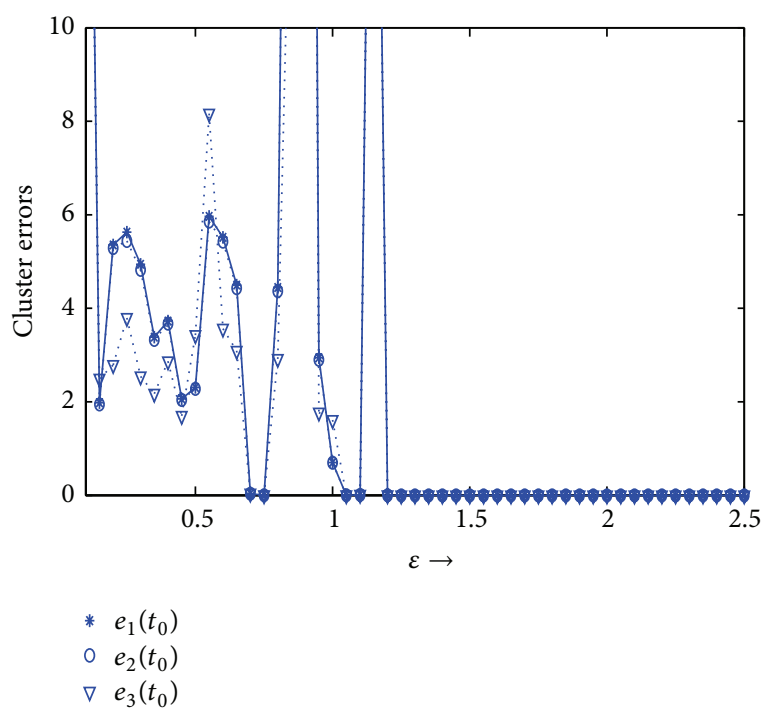

(b) $\theta=5$

FIGURE 3: Dependence of the average cluster errors on the coupling strength $\varepsilon$ for the system (33) with the topology matrix (34). The errors $e_{1}\left(t_{0}\right), e_{2}\left(t_{0}\right)$, and $e_{3}\left(t_{0}\right)$ measure the attractiveness of the manifolds $\mathbb{M}_{K}\left(G_{1}\right), \mathbb{M}_{K}\left(G_{2}\right)$, and $\mathbb{M}_{K}\left(G_{3}\right)$, respectively.

Choose the initial conditions $\left(u_{i}(0), v_{i}(0)\right.$, and $\left.w_{i}(0)\right)$ randomly on $[-1,1] \times[-1,1] \times[-1,1]$, and pick $t_{0}=500$. Fix the coupling weight $\theta$ at 0.2 , and 5 ; the dependence of the cluster errors on $\varepsilon$ is shown, respectively, in Figure 3. As can be seen, there is a good agreement between Figures 3 and 2 .

\section{Conclusions}

In summary, this paper introduced a novel index of partial synchronizability of a network. It is shown that partial synchronization can be ensured by the conditions merely on the quasi-submatrices corresponding to the clusters. If a network is composed of a great mount of nodes, the enormous amount of calculation can be reduced by replacing the 
coupling matrix with several quasi-submatrices. Numerically, different types of partial synchronization occur in a starglobal network when the coupling strength is increased, the order of which is forecasted accurately by our result. It should be a meaningful and effective method to study partial synchronization with different partitions.

In the past decades, the networked control systems have attracted much attention due to their applications covering a wide range of industries. And the network-induced phenomena under consideration in engineering have been discussed widely, including missing measurements [29], fading measurements [30], and probabilistic sensor delays [31]. Therefore, the obtained approach for partial synchronization in this paper might be applicable to the complex networks with networked induced phenomena. The related studies should be one of the future research topics.

\section{Conflict of Interests}

The authors declare that they have no conflict of interests regarding the publication of this paper.

\section{Acknowledgments}

The authors gratefully acknowledge the support of the Natural Science Foundation of Zhejiang Province (no. LQ12A01003), the Natural Science Foundation of Hebei Province in China (no. A2014205152), the Natural Science Foundation of Guangxi Province (no. 2013GXNSFAA019006), and the National Natural Science Foundation of China (nos. 11162004 and 11171084).

\section{References}

[1] L. M. Pecora and T. L. Carroll, "Synchronization in chaotic systems," Physical Review Letters, vol. 64, no. 8, pp. 821-824, 1990.

[2] A. Arenas, A. D. Guilera, J. Kurths, Y. Moreno, and C. Zhou, "Synchronization in complex networks," Physics Reports, vol. 469, no. 3, pp. 93-153, 2008.

[3] W. Yu, G. Chen, and J. Lü, "On pinning synchronization of complex dynamical networks," Automatica, vol. 45, no. 2, pp. 429-435, 2009.

[4] J. B. Zhang, Z. J. Ma, and G. Zhang, "Cluster synchronization induced by one-node clusters in networks with asymmetric negative couplings," Chaos, vol. 23, no. 4, Article ID 043128, 7 pages, 2013.

[5] J. Zhang, Z. Ma, and J. Cao, "Full synchronization studied by a set of partitions connected together," Abstract and Applied Analysis, vol. 2013, Article ID 352826, 8 pages, 2013.

[6] X. Qian, X. Shen, G. Dai, J. Zhang, and C. Lv, "Clapping and broadcasting synchronization in wireless sensor network," in Proceedings of the 6th International Conference on Mobile Adhoc and Sensor Networks (MSN '10), pp. 140-145, Hangzhou, China, December 2010.

[7] J. Hu, Z. D. Wang, H. L. Dong, and H. J. Gao, "Recent advances on recursive filtering and sliding mode design for networked nonlinear stochastic systems: a survey," Mathematical Problems in Engineering, vol. 2013, Article ID 646059, 12 pages, 2013.
[8] J. Zhang, Z. Liu, and J. Xu, "Synchronization in oscillator networks with coupling balance," Chaos, Solitons \& Fractals, vol. 39, no. 2, pp. 556-566, 2009.

[9] L. M. Pecora and T. L. Carroll, "Master stability functions for synchronized coupled systems," Physical Review Letters, vol. 80, no. 10, pp. 2109-2112, 1998.

[10] W. Wu and T. Chen, "Partial synchronization in linearly and symmetrically coupled ordinary differential systems," Physica $D$, vol. 238, no. 4, pp. 355-364, 2009.

[11] W. Sun, R. Wang, W. Wang, and J. Cao, "Analyzing inner and outer synchronization between two coupled discrete-time networks with time delays," Cognitive Neurodynamics, vol. 4, no. 3, pp. 225-231, 2010.

[12] B. Liu, W. Lu, and T. Chen, "New conditions on synchronization of networks of linearly coupled dynamical systems with nonLipschitz right-hand sides," Neural Networks, vol. 25, pp. 5-13, 2012.

[13] J. B. Zhang, Z. J. Ma, and G. R. Chen, "Robustness of cluster synchronous patterns in small-world networks with intercluster co-competition balance," Chaos, vol. 24, no. 2, Article ID 023111, 8 pages, 2014.

[14] L. L. Li and J. D. Cao, "Cluster synchronization in an array of coupled stochastic delayed neural networks via pinning control," Neurocomputing, vol. 74, no. 5, pp. 846-856, 2011.

[15] C. Hu and H. Jiang, "Cluster synchronization for directed community networks via pinning partial schemes," Chaos, Solitons \& Fractals, vol. 45, no. 11, pp. 1368-1377, 2012.

[16] X. Liu and T. Chen, "Cluster synchronization in directed networks via intermittent pinning control," IEEE Transactions on Neural Networks, vol. 22, no. 7, pp. 1009-1020, 2011.

[17] Z. Ma, Z. Liu, and G. Zhang, "A new method to realize cluster synchronization in connected chaotic networks," Chaos, vol. 16, no. 2, Article ID 023103, 9 pages, 2006.

[18] J. Zhang, Z. Ma, and J. Cao, "Hub-induced synchronization in scale-free networks with cluster structure," Abstract and Applied Analysis, vol. 2014, Article ID 149453, 7 pages, 2014.

[19] Z. Ma, S. Zhang, G. Jiang, and K. Li, "Effect of the coupling matrix with a weight parameter on synchronization pattern in a globally coupled network," Nonlinear Dynamics, vol. 74, no. 1-2, pp. 55-64, 2013.

[20] V. N. Belykh, I. V. Belykh, and M. Hasler, "Hierarchy and stability of partially synchronous oscillations of diffusively coupled dynamical systems," Physical Review E: Statistical, Nonlinear, and Soft Matter Physics, vol. 62, no. 5, part A, pp. 6332-6345, 2000.

[21] M. Golubitsky, I. Stewart, and A. Török, "Patterns of synchrony in coupled cell networks with multiple arrows," SIAM Journal on Applied Dynamical Systems, vol. 4, no. 1, pp. 78-100, 2005.

[22] M. Golubitsky and I. Stewart, "Nonlinear dynamics of networks: the groupoid formalism," Bulletin of the American Mathematical Society, vol. 43, no. 3, pp. 305-364, 2006.

[23] V. N. Belykh, I. V. Belykh, and M. Hasler, "Connection graph stability method for synchronized coupled chaotic systems," Physica D: Nonlinear Phenomena, vol. 195, no. 1-2, pp. 159-187, 2004.

[24] C. W. Wu and L. O. Chua, "Synchronization in an array of linearly coupled dynamical systems," IEEE Transactions on Circuits and Systems I: Fundamental Theory and Applications, vol. 42, no. 8, pp. 430-447, 1995.

[25] W. L. Lu and T. P. Chen, "New approach to synchronization analysis of linearly coupled ordinary differential systems," Physica D: Nonlinear Phenomena, vol. 213, no. 2, pp. 214-230, 2006. 
[26] J. F. Heagy, L. M. Pecora, and T. L. Carroll, "Short wavelength bifurcations and size instabilities in coupled oscillator systems," Physical Review Letters, vol. 74, no. 21, pp. 4185-4188, 1995.

[27] I. Belykh, V. Belykh, K. Nevidin, and M. Hasler, "Persistent clusters in lattices of coupled nonidentical chaotic systems," Chaos, vol. 13, no. 1, pp. 165-178, 2003.

[28] X. W. Liu and T. P. Chen, "Boundedness and synchronization of $y$-coupled Lorenz systems with or without controllers," Physica D: Nonlinear Phenomena, vol. 237, no. 5, pp. 630-639, 2008.

[29] J. Hu, Z. Wang, B. Shen, and H. Gao, "Quantised recursive filtering for a class of nonlinear systems with multiplicative noises and missing measurements," International Journal of Control, vol. 86, no. 4, pp. 650-663, 2013.

[30] J. Hu, Z. D. Wang, and H. J. Gao, "Recursive filtering with random parameter matrices, multiple fading measurements and correlated noises," Automatica, vol. 49, no. 11, pp. 34403448, 2013.

[31] J. Hu, Z. Wang, B. Shen, and H. Gao, "Gain-constrained recursive filtering with stochastic nonlinearities and probabilistic sensor delays," IEEE Transactions on Signal Processing, vol. 61, no. 5, pp. 1230-1238, 2013. 


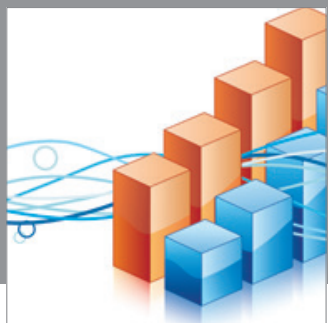

Advances in

Operations Research

mansans

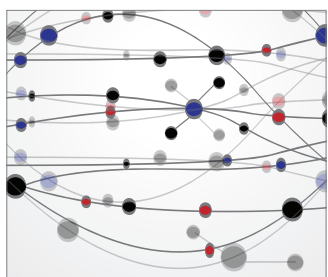

The Scientific World Journal
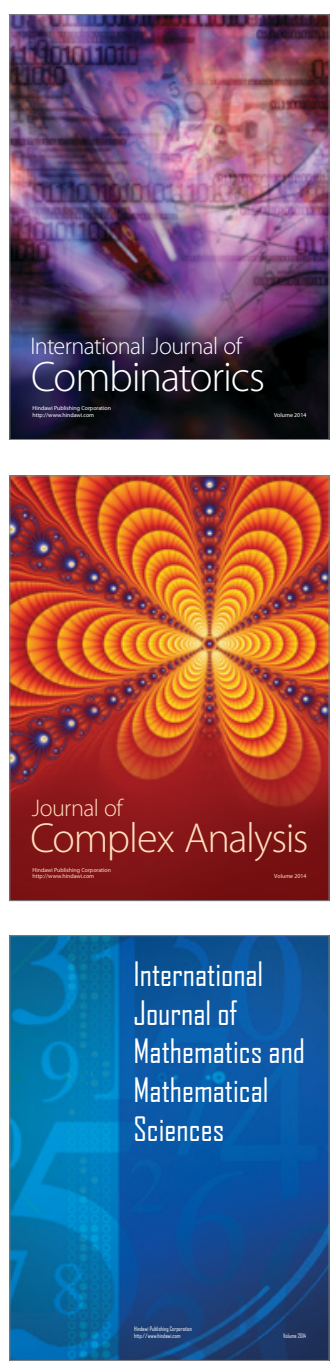
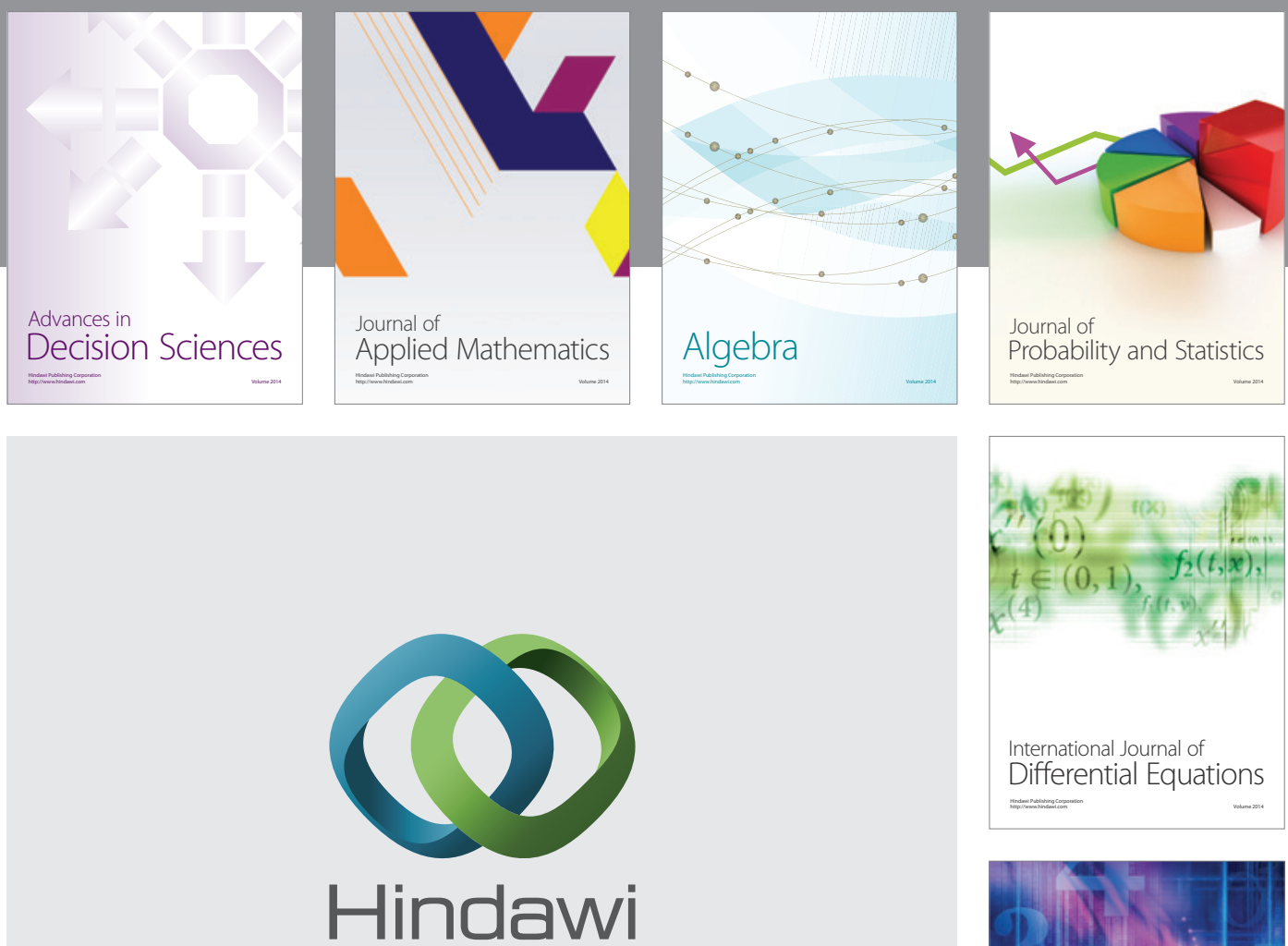

Submit your manuscripts at http://www.hindawi.com
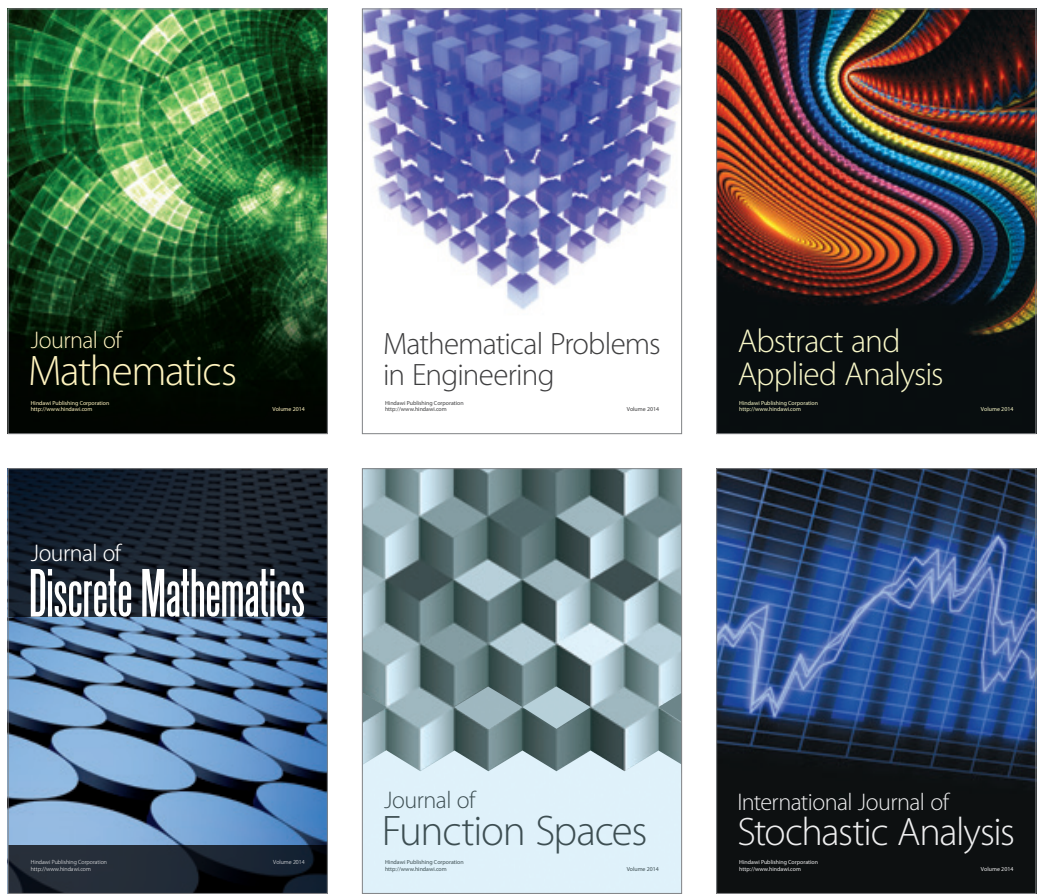

Journal of

Function Spaces

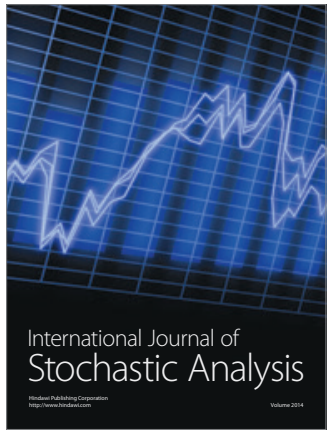

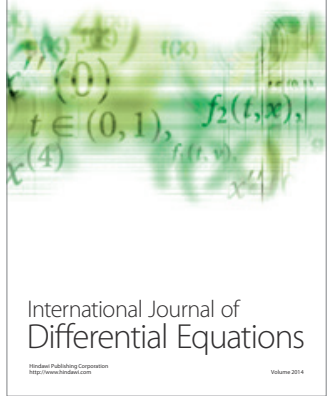
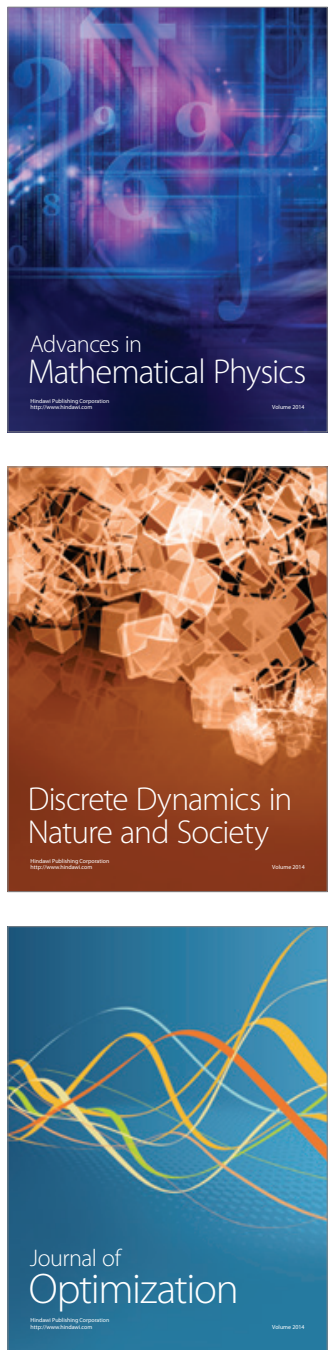